\title{
Reinterpretation of a classic method for the quantification of cell density within biofilms of Listeria monocytogenes
}

\begin{abstract}
Food product contamination by Listeria monocytogenes is mainly related to the pathogen's ability to form biofilms on food processing surfaces. This study aims to correlate a method based on a microplate with a final quantification of the cell density in $\mathrm{CFU} \mathrm{cm} \mathrm{cm}^{-2}$ within the biofilms formed by different strains of $L$. monocytogenes. It is considered that being aware of the capacity that different strains of $L$. monocytogenes possess to form biofilms is highly relevant to address specific cleaning and disinfection procedures in industrial environments. A total of 17 strains with distinct origins and genetic characteristics were employed in this study. Despite all strains were shown to be capable of forming biofilms, significant differences were observed between strains. The data obtained suggests that working with cell counts over a surface is more accurate than absorbance values when regarding biofilms, and therefore our reinterpretation of the classic assay is a quick and manageable method for classifying biofilm producer strains prior to carrying out more complex analyses.
\end{abstract}

Keywords: Listeria monocytogenes, biofilms, quantification, biofilms detection, food hygiene
Volume 6 Issue 2 - 2018

\section{Carolina Ripolles-Avila,Abel G Ríos- Castillo,Alfons E Guerrero-Navarro, José J Rodríguez-Jerez}

Department of Food and Animal Science, Universitat Autònoma de Barcelona, Spain

Correspondence: José J Rodríguez Jerez, Department of Food and Animal Science, Faculty of Veterinary Science, Universitat Autònoma de Barcelona, Spain, Tel +34 9358 I |448, Fax +34 933807558, Email josejuan.rodriguez@uab.cat

Received: December 23, 2017 | Published: March 09, 2018
Abbreviations: BPW, buffered peptone water; CECT, spanish type culture collection; CFU, colony forming units; INIA, national research institute, agricultural and food technology; OD values, optical density values; TSA, tryptone soya agar; TSB, tryptone soya broth; TSYEB, tryptone soya yeast extract broth

\section{Introduction}

Food safety is one of the biggest concerns of food industrial development. ${ }^{1}$ This is mainly related to foodborne diseases, which are the most widespread health problem in the contemporary world and an important cause of reduced economic productivity. ${ }^{2}$ Listeria monocytogenes is an ubiquitous organism naturally present in soil and water, capable of contaminating a wide variety of foods when introduced into food-processing environments due to its hardy growth characteristics. $^{3-5}$ It causes human listeriosis, an infection with a low incidence but a high mortality rate amongst human populations with vulnerable immune systems and the elderly. ${ }^{6}$ This, along with its connection with stillbirth and miscarriage, makes it a considerable public health problem.?

L. monocytogenes is capable of forming biofilms on food processing surfaces. ${ }^{8} \mathrm{~A}$ biofilm is an aggregation of microorganisms growing on a surface and embedded in a matrix of self-produced extracellular polymeric substances which exhibit an altered phenotype as regards growth rate and gene transcription. ${ }^{9-13}$ These biofilms can act as reservoirs for spoilage or pathogenic bacteria and are directly related to product contamination. ${ }^{14,15}$ It has been observed that attachment to surfaces and the subsequent biofilm formation confers resistance on the cells to antimicrobials agents, UV light, desiccation and treatments with sanitising agents, which is not commonly found when the same cell is planktonic. ${ }^{3,16}$ Furthermore, the capability of foodborne bacteria to outlast the stresses that are habitually encountered in food processing such as refrigerating, disinfecting, acidity and salinity, is enhanced by biofilm formation. ${ }^{10,17,18}$
Various direct and indirect observation methods have been used to evaluate the biofilm-forming ability of $L$. monocytogenes. ${ }^{7,9,10,19}$ Direct methods are based on directly observing the microbial colonisation. These methods include microscopy techniques such as light, transmission electron, laser-scanning confocal, scanning electron and epifluorescence microscopy. ${ }^{8}$ Blackman und Frank ${ }^{20}$ observed that not only can biofilm levels be underestimated when epifluorescence microscopy is employed because the thickness is not being measured, but also that the area covered by cells may be overestimated because some extracellular polymer can be stained. Despite this drawback, when studying attached bacteria on surfaces it is important to use direct microscopy to obtain accurate results. ${ }^{21}$ Indirect methods, on the other hand, are those that detach the microorganisms from the surface before counting them. These methods include not only standard plate counts, roll techniques and sonication, but also other procedures that estimate the number of adhered microorganisms in situ by measuring some feature of the adhered organism. ${ }^{22}$ These include techniques such as radio labelled bacteria, enzyme-linked immunosorbent assay, biologic assays, stained bacterial films and microtiter plate procedures. ${ }^{23}$

Focusing on the microtiter plate procedure, there are some assays related to biofilm formation. One of these is the crystal violet method, which was proposed by Christensen et al. ${ }^{24}$ and adapted by others. ${ }^{3,8,25}$ This assay is comprised of growing microbial biofilms on microtiter plates, staining them with crystal violet and then solubilising the bound dye to measure its concentration. It has been extensively employed for microbial culture screening. ${ }^{7,26}$ However, the use of this method only allows the classification of the strains from the most to the lowest biofilm producer, but you do not know the exact number of cells forming the biofilm.

For the food industry, it is completely crucial to be able to detect biofilms formed in food-contact surfaces rapidly in order to assess their hygienic state and take associate decisions regarding to cleaning 
and disinfection procedures. One of the methods that can be employed is the use of biodetectors such as Bio finder that detects the presence of biofilms on surfaces within seconds, allowing the food industry to visually identify if biofilm exist in the food-contact surface. This study aimed to investigate the potential for adapting an existing method that evaluates biofilm production qualitatively to a method that quantifies exactly microbial biomass to polystyrene. The main purpose of this study was to quantify the cell density within biofilms of different strains of L. monocytogenes using crystal violet assay with a further reinterpretation of this classic method to obtain the number of cells forming the biofilm. For the purposes of developing the assay, a correlation curve between Optical Density (OD) values and Colony Forming Units (CFU) per square centimetre was performed. The different $L$. monocytogenes strains were compared in order to investigate their potential as a biofilm formers. Finally, some of the wells on where the biofilm was formed, were observed by microscopy and others, were examined using a biofilm biodetector.

\section{Materials and methods}

\section{Characterisation of isolates}

Five culture collection strains (CECT) and twelve isolates of $L$. monocytogenes were used in this study (sources and serotype are listed in Table 1). ${ }^{27}$ These seventeen different strains were selected for the study as the basis for screening strains that belong to distinct linages. Procedures for collecting, identifying and typifying some of these isolates by serotyping have been previously described. ${ }^{28}$ The different bacterial strains were stored at $4^{\circ} \mathrm{C}$ as frozen-dried cultures, which were then recovered on Buffered Peptone Water (BPW, bioMérieux ${ }^{\circledR}$, Marcy l'Etoile, France), cultivated at $30^{\circ} \mathrm{C}$ for 48 hours, streaked onto Tryptone Soya Agar (TSA) (CM0131, Oxoid, Spain) and cultivated at $30^{\circ} \mathrm{C}$ for 48 hours. Finally, working cultures were kept on TSA slants at $4^{\circ} \mathrm{C}$ to be used within 30 days.

\section{Spectrophotometric determination of the crystal violet dye}

The crystal violet dye solution was prepared at a concentration of $0.8 \%$ in sterile distilled water. One millilitre of this solution was dispensed in a spectrophotometric cell. The absorption spectra for the dye solution was measured by the VIS spectrophotometer type Spectroquant ${ }^{\circledR}$ Prove Spectrophotometer 100, Merck Millipore, USA. The wavelength range to establish the absorbance of the crystal violet solution was set from $320 \mathrm{~nm}$ to $900 \mathrm{~nm}$.

\section{Biofilm formation on the microtiter plates}

Each L. monocytogenes strain was subcultured for 22 hours on TSA and incubated at $37^{\circ} \mathrm{C}$. Biofilm production assays were carried out with a rich undefined medium, TSYEB gluc $1 \%+\mathrm{NaCl} 12 \%$, which consisted of tryptone soya broth (TSB) (CM0129, Oxoid, Spain) supplemented with $0,3 \% \mathrm{w} / \mathrm{v}$ yeast extract $(212750, \mathrm{BD}$, Spain), $1 \%$ $\mathrm{w} / \mathrm{v}$ glucose (4125012, Biolife, Spain) and $2 \% \mathrm{w} / \mathrm{v}$ sodium chloride (131659, Panreac, Spain). The overnight cultures on TSA were transferred by collecting bacterial colonies from the medium surface to $10 \mathrm{ml}$ of $\mathrm{TSYEB}_{\text {glucl } \%+\mathrm{NaCl} 2 \%}$, resulting in a suspension of $10^{6} \mathrm{CFU}$ $\mathrm{ml}^{-1}$, which was then vortexed. This concentration was standardized by photometric readings with a Densimat photometer (bioMérieux ${ }^{\circledR}$, Marcy l'Etoile, France). After vortexing, sixteen volumes per strain of $100 \mu 1$ were transferred into PVC microtiter plate (Sudelab, Spain) wells. The microtiter plates had been previously rinsed in $70 \%$ ethanol and air dried. The microtiter plates were incubated at $30^{\circ} \mathrm{C}$ for 48 hours. Each experiment included sixteen wells of TSYEB ${ }_{\text {gluc } 1 \%+\mathrm{NaCl}_{12} \%}$ medium without L. monocytogenes as control wells.

\section{Biofilm quantification using crystal violet assay}

After 48 hours of incubation, medium from each of the wells was withdrawn into a discard container and the microtiter plate wells were washed twice with $100 \mu \mathrm{l}$ of sterile water to eliminate the unattached cells. The plates were then air dried for 45 minutes. Each well was stained by adding $100 \mu 1$ of $0.8 \%$ crystal violet solution in water and the microtiter plates were incubated for 45 minutes at room temperature. After staining, the unbound dye was eliminated and the biofilms were visible as purple rings formed at the base of the well (Figure 1). In order to proceed with the quantitative analysis of the biofilm, the crystal violet was solubilised by adding $150 \mu 1$ of $95 \%$ ethanol to fade the wells and was incubated for 30 minutes at room temperature. $100 \mu 1$ from each of these wells was transferred to a new sterile polystyrene microtiter plate and the crystal violet level present in the destaining solution was measured by a microtiter plate reader (340 ATTC, SLT Labinstruments, Vienna - Austria) at an optical density of $595\left(\mathrm{OD}_{595}\right)$. A procedure was performed to correlate the level of crystal violet dye with the number of bacteria stained in the

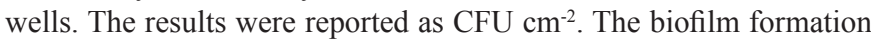
on the polystyrene plates and its subsequent quantification was carried out three times on three different days for all the strains of $L$. monocytogenes. The averages and standard deviations were calculated for all repetitions of the experiment.

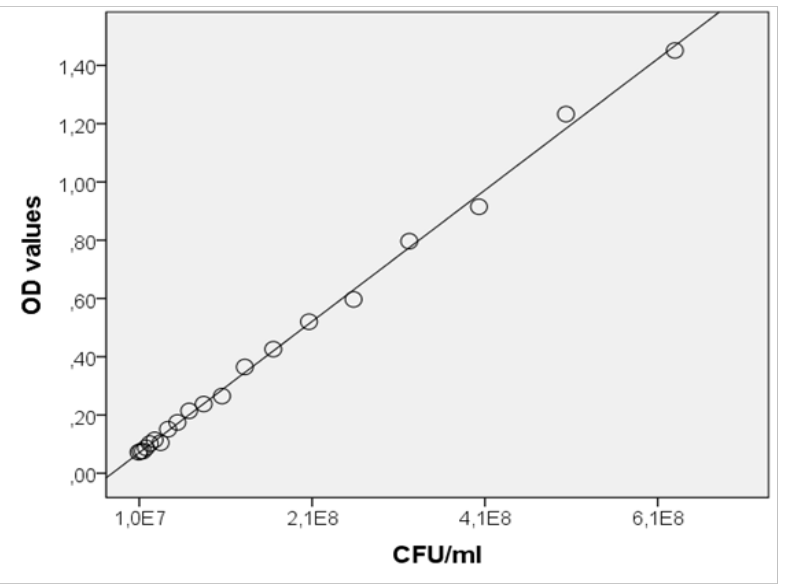

Figure I Correlation between the absorbance of crystal violet in OD values and the colony-counting method. Each value corresponds to the mean of three replicates.

\section{Correlation curve between OD values and CFU $\mathrm{ml}^{-1}$}

The correlation curve was obtained for the strain of L. monocytogenes CECT 935, which is an international reference of ISO $11290^{29}$ and for three more strains L. monocytogenes A7, EDG-e, CECT 5366, which were randomly chosen in order to investigate if there were differences between strains. To create the correlation curve, the overnight cultures on TSA were transferred by looping bacterial colonies to $10 \mathrm{ml}$ of $\mathrm{TSYEB}_{\text {gluc } 1 \%+\mathrm{NaCl} 12 \%}$, resulting in a suspension of $10^{9} \mathrm{CFU} \mathrm{ml} l^{-1}$. This was vortexed and one millilitre was then transferred to an Eppendorf tube (Eppendorf, Hamburg, Germany). The samples were centrifuged by the Heraeus Pico 17 Centrifuge, Thermo Scientific for 30 seconds at $12000 \mathrm{rcf}$. In the inoculated Eppendorf tubes, L. monocytogenes was 
visible as a bacterial pellet. The supernatant was discharged and the bacterial pellets were washed twice with sterile water. Each Eppendorf tube was stained by adding $1 \mathrm{ml}$ of $0.8 \%$ crystal violet solution and incubated for 45 minutes at room temperature. After staining, the unbound dye was eliminated and the pellets were stained purple. The crystal violet was solubilised by adding $1 \mathrm{ml}$ of $95 \%$ ethanol, which destained the pellets, and was then incubated for 30 minutes. Serial dilutions of this solution with ethanol were prepared to observe absorbance from high to low (Figure 2). $100 \mu$ l was transferred from each Eppendorf tube to a polystyrene microtiter plate and the crystal violet level was measured in a microtiter plate reader at an optical density of $595 \mathrm{~nm}$. The optical density values were correlated with the CFU ml-1 obtained by plating on TSA from the initial bacterial suspension.

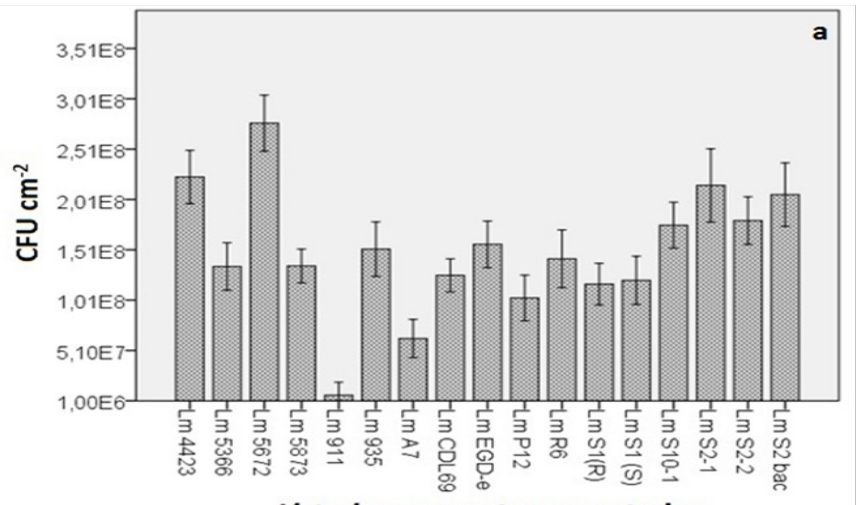

Listeria monocytogenes strains

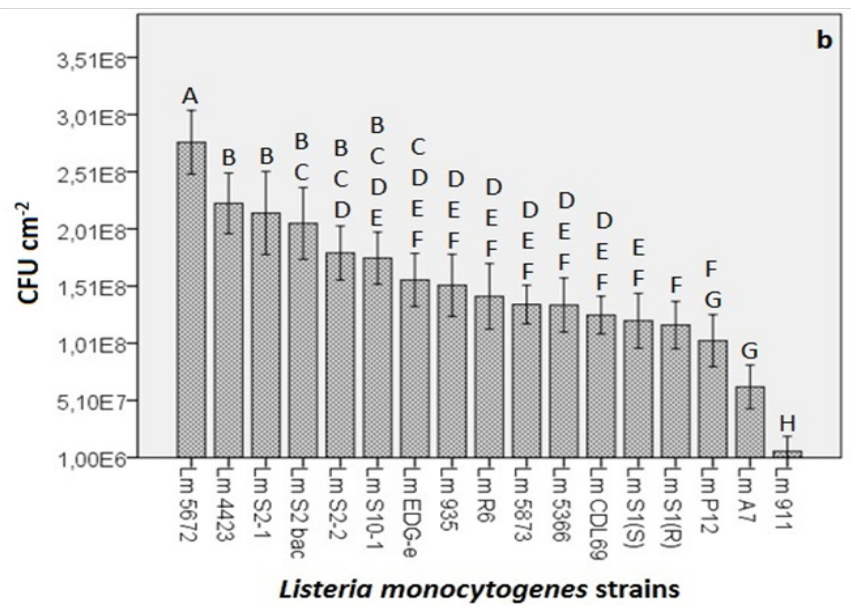

Figure 2 (A) Microbial counts obtained from the quantification of the biofilm formation of different strains of $L$. monocytogenes $(\mathrm{Lm})$. In the graphs, the $y$-axis shows the CFU $\mathrm{cm}^{-2}$ and $\mathrm{x}$-axis shows the different strains employed in the study. Each value corresponds to the mean of three replicates. The bars represent the standard error of the mean (B) Strains sorted in descending order from the most major biofilm-producing strain to the least. The graph shows the eight different groups obtained through the Tukey's B test where significance was set at $\mathrm{p}<0.05$.

\section{Detection of biofilms formed on microtiter plates using a biofilm detector (Biofinder)}

The biofilms were formed as described in section 2.3. After a 48-hour incubation period, medium from each of the wells was withdrawn into a discard container and the microtiter plate wells were washed twice with $100 \mu \mathrm{l}$ of sterile water to eliminate the unattached cells. One drop of Biofinder (iTram Higiene, Vic, Spain) was then introduced into the wells containing the 17 different strains of $L$. monocytogenes. The reaction was observed after 10 seconds.

\section{Microscopic observation of the biofilm formation}

L. monocytogenes CECT 935 was the only strain used for this evaluation. The biofilms were formed in the same microtiter plates and stained using the method described in section 2.4. After the biofilms were stained, the wells of the microtiter plate were sliced for observation by microscopy. The main objective was to qualitatively compare the biofilms formed at 48 hours with the biofilms formed after 7 days of incubation. To form the one week incubated biofilms, volumes of $100 \mu \mathrm{l}$ of $L$. monocytogenes CECT 935 inoculum were transferred into sixteen PVC microtiter plates and incubated for seven days at $30^{\circ} \mathrm{C}$ with different washes and renewal of the culture medium at 48-72-24-24 hours of incubation in order to remove the unattached cells and promote the growth of the adhered cells. The washes and renewal of nutrients was done by washing three times with sterile distilled water and $100 \mu 1$ of new sterile TSYEB $_{\text {gluc } 1 \%+\mathrm{NaCl} 2 \%}$ medium was dispensed into the previously incubated wells.

\section{Statistical analysis and reproducibility of results}

Sixteen replicates for each of the three independent repeats were carried out for each trial. The data presented are the means of the data generated from the three independent experiments. The data from all the experiments were analysed by means of the SPSS statistical package using the ANOVA general linear model with subsequent analysis of variance and Tukey's B test. A $p$ value of $<0.05$ was considered to be significant.

\section{Results and discussion \\ Spectrum of crystal violet dye}

The absorption spectrum of the $0.8 \%$ crystal violet solution was performed not only to investigate where the maximum absorption peak was, but also to see if it concurred with the previously published literature. The maximum absorption peak was observed at $595 \mathrm{~nm}$ and therefore this was the wavelength employed in the study. The results were consistent with the published literature on where preliminary studies using microtiter plates for assessing L. monocytogenes biofilm formation had used the same wavelength. ${ }^{3,8,30}$

\section{Growth of L. monocytogenes on TSYEB ${ }_{\text {gluc } 1 \%+\mathrm{NaCl} 2 \%}$}

Seventeen different $L$. monocytogenes strains were tested using a modified crystal violet assay for their quantification. The evaluation of their biofilm-forming ability was carried out after a 48-hour incubation period at $30^{\circ} \mathrm{C}$. The media employed was the one proposed by Pan et al., ${ }^{31}$ which was TSYEB with a supplement of glucose and sodium chloride.

L. monocytogenes is an ubiquitous organism that can grow in a large variety of environments using different nutrient sources. ${ }^{32}$ Preceding studies have shown that the growth and subsequent biofilm formation of L. monocytogenes is affected by the growth media used. ${ }^{33}$ Pan et al., ${ }^{31}$ demonstrated that sodium chloride and glucose concentrations play a crucial role in the formation of biofilms. In his study, TSYEB ${ }_{\text {gluc } 1 \%+\mathrm{NaCl} 12 \%}$ medium was employed as a growth media and almost all the $L$. monocytogenes strains exhibited enhanced biofilm formation at $30^{\circ} \mathrm{C}$ when $2 \%$ salt and $1 \%$ glucose concentrations were added. Hence, TSYEB with glucose and sodium chloride was chosen for the study. For developing the assay with optimum conditions, 
apart from the growth medium, growth temperature as an influential parameter for the biofilm formation was also assessed. The formation of biofilms was much lower at temperatures of $12^{\circ} \mathrm{C}$ and $22^{\circ} \mathrm{C}$ than at $30^{\circ} \mathrm{C}$ (data not shown), so $30^{\circ} \mathrm{C}$ was the temperature selected for this research study.

\section{Correlation curve for the biofilm quantification}

The method was initially proposed by Christensen et al., ${ }^{24}$ and adapted by other authors. ${ }^{3,8,25}$ With the reinterpretation suggested in this study, however, the results will be obtained as microbial counts which reflects more accurately the biomass of the formed biofilms. To achieve this, a correlation between $\mathrm{OD}_{595}$ values and $\mathrm{CFU} \mathrm{ml}{ }^{-1}$ was performed to set up a correlation curve as a basis for comparing the $\mathrm{OD}_{595}$ values from the experiments with estimates of the numbers of bacteria represented by these $\mathrm{OD}_{595}$ values, as other authors have done. ${ }^{34}$ The measured data on the relationship between $\mathrm{OD}$ values and $\mathrm{CFU} \mathrm{ml^{-1 }}$ for L. monocytogenes CECT 935 is shown in Figure 1. The results indicate that the method is enormously reliable for quantifying $L$. monocytogenes organisms. A curve representing the relationship between OD values and the corresponding cells counts was plotted and analysed using linear regression. The bacterial cells showed a linear relationship in the range of $1 \times 10^{7}-\mathrm{x} 10^{9}$ cells $\mathrm{ml}^{-1}$ and the correlation between the two methods was high (coefficient of determination, $\mathrm{R}^{2}=0.998$ ). Any statistically differences were found between the correlation curves performed using the four strains of $L$. monocytogenes and therefore it was proved that the same correlation curve can be used to estimate the number of the cells forming the biofilms of the seventeen different strains of $L$. monocytogenes. In addition, obtaining the results as microbial counts is a better and more useful tool for simultaneously comparing different quantification methods.

\section{Quantification of the biofilm formation on polystyrene microtiter plates}

The biofilm formation was investigated statically after a 48-hour incubation period. Under these conditions, the $\mathrm{OD}_{595}$ values of crystal violet obtained from the faded biofilm varied greatly for the different strains of L. monocytogenes. Using the standard curve shown in Figure 1 and correcting the value to obtain $\mathrm{cm}^{2}, \mathrm{CFU} \mathrm{cm} \mathrm{cm}^{-2}$ readings ranging from $10^{8}$ to $10^{6}$ were observed (Figure $2 \mathrm{~A}$ ).

When the Tukey's B test was applied, 8 different groups (codified from $\mathrm{A}$ to $\mathrm{H})$ with significant differences $(p<0.05)$ between them were found (Figure 2B). In those groups, there were strains that belonged to a single group (e.g. Lm 5672, 4423, S2-1, S1(R), A7 and 911) and others that could be classified into more than just one group. For example, strain Lm 5672 fits into group A only, whilst strain Lm S2 ${ }^{\text {bac }}$ fit into groups B and C.

All of the different strains of $L$. monocytogenes were found to be capable of forming biofilms, although some of the strains were considered to be better biofilm formers on the basis of the difference in the microbial counts (e.g. $2 \log$ difference between the strongest and the weakest biofilm producer strain). L. monocytogenes CECT 5672 , categorised in group A, was the strongest biofilm producer and was significantly different $(p<0.05)$ from the rest of the strains. $L$. monocytogenes CECT 911, categorised in group H, was the weakest biofilm producer with the minimum cell count level detected and significant differences $(p<0.05)$ from the rest of the strains were also observed. L. monocytogenes 4423, S2-1, S2 ${ }^{\text {bac }}, \mathrm{S} 2-2$ and S10-1 were categorised in group B, meaning that no significant differences ( $p=0.298)$ were observed among them. With the rest of the strains, however, significant differences $(p<0.05)$ were found, except for $L$. monocytogenes $\mathrm{S} 2^{\text {bac }}, \mathrm{S} 2-2$ and $\mathrm{S} 10-1$, which were categorised in more than one group. For example, in the case of L. monocytogenes $\mathrm{S}^{\text {bac }}$, not only were no significant differences observed among the strains that belonged to group $\mathrm{B}$, but no significant differences $(p=0.06)$ were noticed with L. monocytogenes S2-2, S10-1 and EDG-e either (group C). The most similar strains regarding their capacity to produce biofilms were thought to be the following: L. monocytogenes EDG-e, R6, CDL69 and CECT 935, 5873, 5366 which were categorised simultaneously in three groups D, E and F. These strains presented less significant differences when compared with the rest of strains, thus they were considered to share more similarities.

The seventeen strains employed in this study belonged to different serotypes (see Table 1 in chapter 2.1), which can be grouped into divisions. Division I consists of serotype $4 \mathrm{~b}$, Division II consists of serotypes $1 / 2 \mathrm{a}$ and $1 / 2 \mathrm{c}$ and strains belonging to serotypes $2 \mathrm{a}$ are considered as other serotypes. Variations in biofilm production at the level of divisions was also observed, but there were no significant differences. In contrast, other studies have found statistically significant differences when correlating the formation of biofilms with phylogenetic division. ${ }^{3,8}$ This can be ascribed to the material employed as varying adhesion between different lots of microtiter plates has been reported. ${ }^{22}$

Table I Listeria monocytogenes isolates used in this study

\begin{tabular}{|c|c|c|}
\hline Isolates & Serovar & Origin* \\
\hline 4423 & $1 / 2 a$ & Ortiz et al.. ${ }^{28}$ \\
\hline 5366 & $4 \mathrm{~b}$ & CECT \\
\hline 5672 & $4 b$ & CECT \\
\hline 5873 & $\mathrm{I} / 2 \mathrm{a}$ & CECT \\
\hline 911 & $1 / 2 c$ & CECT \\
\hline 935 & $4 \mathrm{~b}$ & CECT \\
\hline A7 & $\mathrm{I} / 2 \mathrm{a}$ & INIA \\
\hline CDL69 & $\mathrm{I} / 2 \mathrm{a}$ & Ortiz et al., ${ }^{28}$ \\
\hline EGD-e & $\mathrm{I} / 2 \mathrm{a}$ & Ortiz et al.. ${ }^{28}$ \\
\hline $\mathrm{PI} 2$ & $1 / 2 a$ & INIA \\
\hline R6 & $1 / 2 a$ & INIA \\
\hline $\mathrm{SI}(\mathrm{R})$ & $\mathrm{I} / 2 \mathrm{a}$ & INIA \\
\hline SI(S) & $\mathrm{I} / 2 \mathrm{a}$ & INIA \\
\hline SIO-I & $2 a$ & Ortiz et al.. ${ }^{28}$ \\
\hline S2-I & $\mathrm{I} / 2 \mathrm{a}$ & INIA \\
\hline S2-2 & $\mathrm{I} / 2 \mathrm{a}$ & INIA \\
\hline $\mathrm{S} 2^{\mathrm{bac}}$ & $1 / 2 a$ & Ortiz et al.., ${ }^{27}$ \\
\hline
\end{tabular}

*The different isolates were obtained from Spanish Type Culture Collection (CECT) and the National Research Institute,Agricultural and Food Technology (INIA). From Ortiz et al., ${ }^{28}$ and Ortiz et al.., ${ }^{27}$ the isolates were collected during a three-year study in an Iberian pork processing plant.

\section{Detection of biofilms formed on the microtiter plates using Biofinder}

Biofilm formation detection of the different strains on polystyrene surfaces was also assayed using the product Biofinder. The results (Figure 3 ) showed not only a strong positive reaction with plentiful bubble formation in the different wells, but they also demonstrated that the intensity of the bubbles was higher in the strains that were 
found to be more biofilm-producing than the ones that were less biofilm-producing. The present study demonstrated prolific bubble formation, which was considered to be an intense and positive reaction. Moreover, the use of this product also allowed us to screen the bacterial strains from the strongest biofilm producer to the weakest. The results concurred with those obtained when quantifying the biofilms formed with the different strains of L. monocytogenes, whereas this product can also be used as a screening tool to identify which strains are stronger and weaker biofilm producers.

\section{Microscopic observation of crystal violet stained biofilms}

A qualitative study was conducted to observe not only the typical morphology of the biofilms, but also to show how they increase over the time (Figure 4). The percentage of covered areas at 48 hours of incubation was lower than the percentage after one week of incubation. Figure 4B shows compact biofilm agglomerations with high cell density, as opposed to Figure 4A, which presents a more distributed network of microcolonies all over the polystyrene surface. This is important for visually determining the optimal biofilm network and for further protocols to develop more agglomerated biofilms. For further research, biofilms should be developed under an incubation period of one week due to they showed more compact agglomeration and a higher cell density, which can helps us to better understand biofilm behaviour in its mature stage.

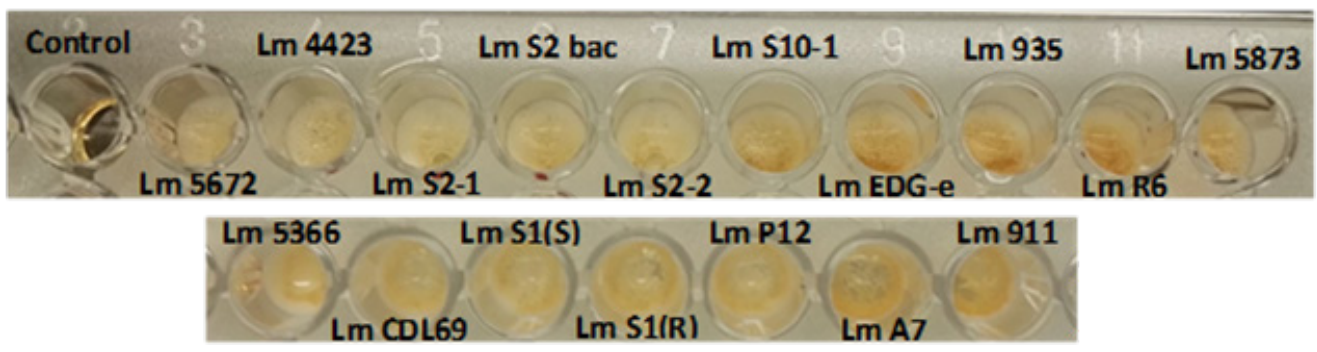

Figure 3 Application of the biodetector to the seventeen strains after biofilm formation. It includes a control well for observing negative reaction.

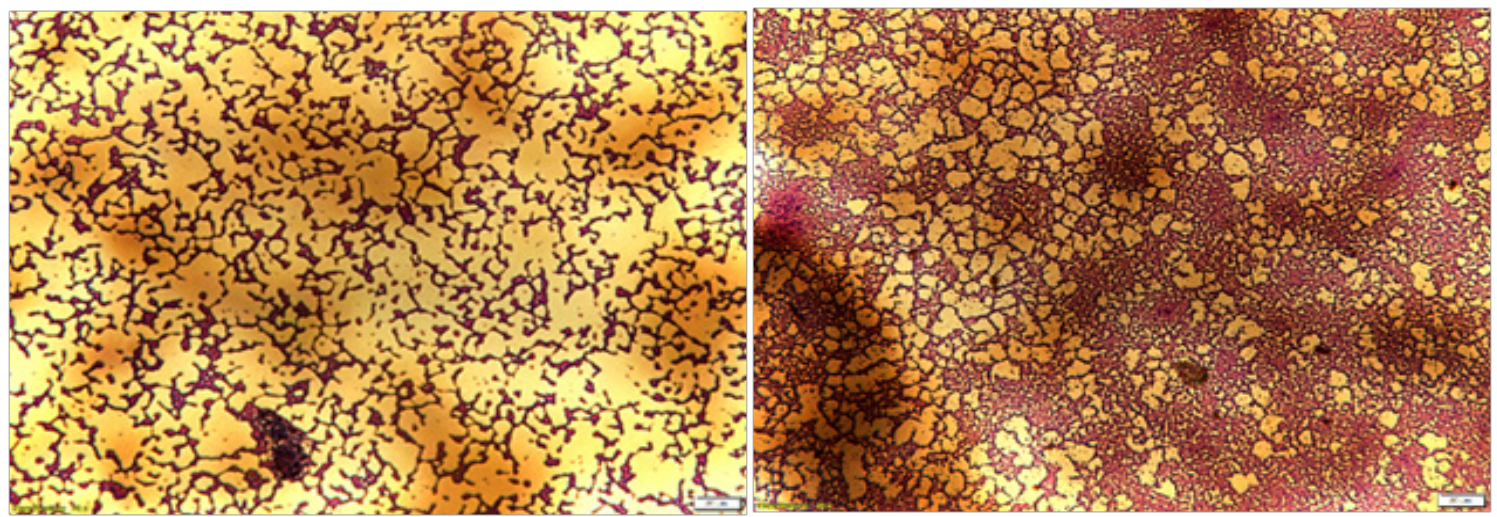

A

Figure 4 Crystal violet stain of L. monocytogenes strain CECT 935 in the wells of the microtiter plate. The images show biofilm formation after incubation periods of 48 hours, I week. Magnification: $20 \mathrm{X}$.

\section{Conclusion}

Crystal violet assay is a rapid and effective method to analyse bacterial adhesion and it allows different parameters to be tested within the same experiment. For the researcher this means a quick, previous step for screening and evaluating different test conditions. Based on the results obtained and the literature survey, it can be concluded that the reinterpretation of this classic method is useful due to it makes quantification as microbial counts possible, it makes comparison with other methods to be easier and it gives more accurate results. Further research is needed to explore the use of this method to rapidly quantify biofilm production on other materials. Extrapolating and adapting the technique to assess the biofilm-forming ability of different bacterial strains could be immediately advantageous in that it means finally having less labour-intensive quantification techniques.

\section{Acknowledgements}

The authors thank Dolors Busquets enormously for her technical assistance. This study was supported by Research Project grants RTA2014-00045-C03-03 (INIA) from the Spanish Ministry of Economy and Competitiveness. The support of the company Itram Higiene (Vic, Barcelona, Spain) is also gratefully acknowledged.

\section{Conflict of interest}

The author declares no conflict of interest.

\section{References}

1. Adams MR, Moss MR. Food Microbiology. In: Thomas Graham House, editor. Guildford, UK: The Royal Society of Chemistry. 2008:1-478.

2. WHO. WHO Commission on Health and Environment. Report of the Panel on Food and Agriculture. Switzerland: WHO, 1992. 191 p.

3. Borucki MK, Peppin JD, White D, et al. Variation in biofilm formation among strains of Listeria monocytogenes. Appl Environ Microbiol. 2003;69(12):7336-7342. 
4. Farber JM, Peterkin PI. Listeria monocytogenes, a food-borne pathogen. Microbiol Rev. 1991;55(3):476-511.

5. Jemmi T, Stephan R. Listeria monocytogenes: a food-borne pathogen and hygiene indicator. Rev Sci Tech Int Epiz. 2006;25(2):571-580.

6. Lund BM, O'Brien SJ. The Occurrence and Prevention of Foodborne Disease in Vulnerable People. Foodborne Pathog Dis. 2011;8(9):961973.

7. Lourenço A Rego F, Brito L, Frank J. Evaluation of Methods To Assess the Biofilm-Forming Ability of Listeria monocytogenes. J Food Prot. 2012;75(8):1411-1417.

8. Djordjevic D, Wiedmann M, McLandsborough LA. Microtiter plate assay for assessment of Listeria monocytogenes biofilm formation. Appl Environ Microbiol. 2002;68(6):2950-2958.

9. Barbosa J, Borges S, Camilo R, et al. Biofilm formation among clinical and food isolates of Listeria monocytogenes. International Journal of Microbiology. 2013;6.

10. Giaouris E, Heir E, Hébraud M, et al. Attachment and biofilm formation by foodborne bacteria in meat processing environments: Causes, implications, role of bacterial interactions and control by alternative novel methods. Meat Scien J. 2014;97(3):289-309.

11. Kalmokoff ML, Austin JW, Wan XD, et al. Adsorption, attachment and biofilm formation among isolates of Listeria monocytogenes using model conditions. J Appl Microbiol. 2001;91(4):725-734.

12. Kretli L, Dietary W. Microbial Biofilms: The Challenge of Food Industry. Biochem Mol Biol J. 2015;1:1-3.

13. Rieu A, Briandet R, Habimana O, et al. Listeria monocytogenes EGD-e biofilms: No mushrooms but a network of knitted chains. Appl Environ Microbiol. 2008;74(14):4491-4497.

14. Brackett RE. Shelf stability and safety of fresh produce as influenced by sanitation and disinfection. J Food Prot. 1992;55(10):808-814.

15. Sommer P, Martin-Rouas C, Mettler E. Influence of the adherent population level on biofilm population, structure and resistance to chlorination. Food Microbiol. 1999;16(5):503-515.

16. Chavant P, Gaillard-Martinie B, Hébraud M. Antimicrobial effects of sanitizers against planktonic and sessile Listeria monocytogenes cells according to the growth phase. FEMS Microbiol Lett. 2004;236(2):241248 .

17. Brooks JD, Flint SH. Biofilms in the food industry: Problems and potential solutions. International Journal of Food Science \& Technology. 2008;43(12):2163-2176.

18. Olsen I. Biofilm-specific antibiotic tolerance and resistance. Eur J Clin Microbiol Infect Dis 2015;34(5):877-886.

19. Silva S, Teixeira P, Oliveira R, et al. Adhesion to and viability of Listeria monocytogenes on food contact surfaces. J Food Prot. 2008;71(7):1379_ 1385 .
20. Blackman IC, Frank JF. Growth of Listeria monocytogenes as a biofilm on various food-processing surfaces. J Food Prot. 1996;59(8):827-831.

21. Lad TL, Costerton JW. Methods for studying biofilm bacteria. J Microbiol Methods. 1990;22:285-307.

22. An YH, Friedman RJ. Handbook of bacterial adhesion: principles, methods, and applications. USA: Humana Press; 2000.

23. Wu H, Zeng M, Fives-Taylor P. The Glycan Moieties and the N-Terminal Polypeptide Backbone of a Fimbria-Associated Adhesin, Fap1, Play Distinct Roles in the Biofilm Development of Streptococcus parasanguinis. Infect Immun. 2007;75(5):2181-2188.

24. Christensen GD, Simpson WA, Younger JJ, et al. Adherence of coagulasenegative staphylococci to plastic tissue culture plates: A quantitative model for the adherence of staphylococci to medical devices. $J$ Clin Microbiol. 1985;22(6):996-1006.

25. Stepanovi S, Vukovic D, Hola V, et al. Quantification of biofilm in microtiter plates: Overview of testing conditions and practical recommendations for assessment of biofilm production by staphylococci. Apmis. 2007;115(8):891-899.

26. O'Toole GA, Kolter R. Initiation of biofilm formation in Pseudomonas fluorescens WCS365 proceeds via multiple, convergent signalling pathways: A genetic analysis. Mol Microbiol. 1998;28(3):449-461.

27. Ortiz S, López V, Martínez-Suárez JV. The influence of subminimal inhibitory concentrations of benzalkonium chloride on biofilm formation by Listeria monocytogenes. Int J Food Microbiol. 2014c;189:106-112.

28. Ortiz S, López V, Martínez-Suárez JV. Control of Listeria monocytogenes contamination in an Iberian pork processing plant and selection of benzalkonium chloride-resistant strains. Food Microbiol. 2014b;39:8188

29. Anonymous. Microbiology of food and animal feeding stuffs - Horizontal method for the detection and enumeration of Listeria monocytogenes, 2008.

30. Harvey J, Keenan KP, Gilmour A. Assessing biofilm formation by Listeria monocytogenes strains. Food Microbiol. 2007;24(4):380-392.

31. Pan Y, Breidt F, Gorski L. Synergistic effects of sodium chloride, glucose, and temperature on biofilm formation by Listeria monocytogenes Serotype 1/2a and 4b Strains. Appl Environ Microbiol 2010;76(5):14331441.

32. Rocourt J, Ben Embarek P, Toyofuku H, et al. Quantitative risk assessment of Listeria monocytogenes in ready-to-eat foods: The FAO WHO approach. FEMS Immunol Med Mic. 2003;35(3):263-267.

33. Poulsen LV. Review: "Listeria monocytogenes in Food Processing. Microbial Bio. 1999;326:321-326.

34. Dong-ju K. Relation of microbial biomass to counting units for Pseudomonas aeruginosa. Afr J Microbiol Res. 2012;6(21):4620-4622. 